\title{
Robust stability properties of soft constrained MPC
}

\author{
Melanie N. Zeilinger, Colin N. Jones and Manfred Morari \\ Automatic Control Laboratory, ETH Zurich, Physikstrasse 3, ETL I 28, CH- 8092 Zurich, Switzerland \\ zeilinger|cjones | morari econtrol.ee.ethz.ch
}

\begin{abstract}
In Model Predictive Control, the enforcement of hard state constraints can be overly conservative or even infeasible, especially in the presence of disturbances. This work presents a soft constrained MPC approach that provides closedloop stability even for unstable systems. Two types of soft constraints are employed: state constraints along the horizon are relaxed by the introduction of two different types of slack variables and the terminal constraint is softened by moving the target from the origin to a feasible steady-state. The proposed method significantly enlarges the region of attraction and preserves the optimal behavior whenever all state constraints can be enforced. Asymptotic stability of the nominal system under the proposed control law is shown, as well as input-tostate stability of the system under additive disturbances and the robust stability properties are analyzed.
\end{abstract}

\section{INTRODUCTION}

Model Predictive Control (MPC) is a control technique that is widely applied for the control of constrained systems. The control action is obtained by solving a constrained finite horizon optimal control problem for the current state of the plant at each sampling time. In a control system, there are generally two types of constraints: constraints on the control input originating from physical limitations of the actuators and constraints on the states or outputs, which represent desired or critical bounds related e.g. to safety or particular system specifications. While input constraints can therefore never be exceeded and are considered as hard constraints, state or output constraints can usually not be enforced at all times in practice, e.g. because of disturbances that are acting on the system.

In this work we propose a soft constrained MPC approach for linear systems that provides stability even for unstable systems. Soft constrained MPC approaches are based on the idea that, due to the nature of the state constraints, violation can often be tolerated for short time periods. Several methods for the development of controllers that enforce state constraints when they are feasible and allow for possible relaxation when they are not have been studied in the literature, see e.g. [12] for an overview. In [16] a simple stabilizing strategy for infinite horizon MPC is proposed that can be applied to both stable and unstable systems. The authors in [21] prove stability of infinite horizon MPC for systems with eigenvalues in the closed unit disk. In [3] the use of $l_{1}$ and $l_{2}$ penalties for constraint violation is compared and it is shown that $l_{1}$ penalties preserve the stability characteristics of the corresponding hard-constrained problem wherever the state constraints can be enforced. A comparison between soft constrained and minimum-time approaches in provided [18]. A soft constrained method for stochastic MPC is developed in [15].

In contrast to soft constrained MPC, robust MPC methods design the control problem for an expected worst-case bound on the disturbance in order to ensure constraint satisfaction and robust stability, see e.g. [10], [13] for an overview. The results can however be conservative since the guarantees are only valid if the disturbance never exceeds the expected bound, requiring a conservative choice of the considered disturbance set.

The proposed method is based on a finite horizon MPC setup and uses a terminal weight as well as a terminal constraint. All input constraints are hard constraints and state constraints are softened in two ways. The terminal constraint is relaxed by allowing the origin to move to any feasible steady-state. All other state constraints are softened by the introduction of two types of slack variables, which is a crucial item for proving stabiliy. Quadratic and $l_{1}$ or $l_{\infty}$ penalties for the constraint violations are introduced in the cost in order to allow for more flexibility in the problem formulation. The use of $l_{1}$ or $l_{\infty}$ penalties allows for exact penalty functions which preserve the optimal MPC behavior whenever the state constraints can be enforced.

We show that in contrast to existing soft constrained MPC schemes asymptotic stability of the nominal system in the absence of disturbances is guaranteed even for unstable systems. The presented approach offers an enlarged region of attraction due to the constraint relaxation that, by choosing the prediction horizon accordingly, can cover any polytopic region of interest up to the maximum stabilizable set for the input-constrained system, i.e. all initial states for which there exists a feasible input at all times such that the state converges to the origin. The robust stability properties of the proposed soft constrained scheme are analyzed and input-tostate stability under additive disturbances is proven. A main advantage of the presented method is that, while stability is formally guaranteed in a robust invariant set that depends on the considered disturbance size, the control law is defined everywhere in the region of interest. In contrast, when using a robust MPC method the control law is only defined for a set of tightened constraints that is determined by the considered disturbance size.

A numerical example demonstrates the soft constrained 
procedure and shows that the constraint relaxation enlarges the robust invariant set where stability can be guaranteed for various disturbance sizes and large disturbances can be tolerated.

The outline of the paper is as follows: In Section III the soft constrained MPC problem is introduced together with its properties. Section IV shows that the proposed control law is optimal wherever the state constraints can be enforced. Asymptotic stability of the nominal system under the proposed control law is then shown in Section V. Section VI analyzes the robustness properties of the proposed scheme and proves input-to-state stability of the uncertain system under the nominal control law. Finally, the properties and advantages of the presented soft constrained MPC approach are illustrated using a numerical example.

\section{NotAtion \& PRELIMINARIES}

A polyhedron is the intersection of a finite number of halfspaces $P=\{x \mid A x \leq b\}$ and a polytope is a bounded polyhedron. If $A \in \mathbb{R}^{m \times n}$ then $A_{i} \in \mathbb{R}^{n}$ is the vector formed by the $i$-th row of $A$. If $b \in \mathbb{R}^{m}$ is a vector then $b_{i}$ is the $i$-th element of $b$. Given a sequence $\mathbf{u} \triangleq\left[u_{0}, \cdots, u_{N-1}\right]$, $u_{j}$ denotes the $j$-th element of $\mathbf{u}$. If a sequence depends on a parameter denoted by $\mathbf{u}(x), u_{j}(x)$ denotes its $j$-th element. If $x \in \mathbb{R}^{n}$ is a vector then $\|x\|_{Q}^{2}=x^{T} Q x$ and $[x]_{+}=\max \{0, x\}$ taken elementwise.

A function $\gamma: \mathbb{R}_{\geq 0} \rightarrow \mathbb{R}_{\geq 0}$ is of class $\mathcal{K}$ if it is continous, strictly increasing and $\gamma(0)=0$ [20]. A function $\beta: \mathbb{R}_{\geq 0} \times \mathbb{R}_{\geq 0} \rightarrow \mathbb{R}_{\geq 0}$ is of class $\mathcal{K} \mathcal{L}$ if, for each fixed $t \geq 0, \beta(\cdot, t)$ is of class $\mathcal{K}$, for each fixed $s \geq 0, \beta(s, \cdot)$ is non-increasing and $\beta(s, t) \rightarrow 0$ as $t \rightarrow \infty$ [20].

Consider the discrete-time linear system

$$
x^{+}=A x+B u,
$$

where $x \in \mathbb{R}^{n}$ is the current state and $u \in \mathbb{R}^{m}$ is the current control input. The solution of system (1) at sampling time $k$ for the initial state $x(0)$ and a sequence of control inputs $\mathbf{u}$ is denoted as $\phi(k, x(0), \mathbf{u})$.

While the system may be unstable, it is assumed to satisfy the following assumption:

Assumption II.1. The pair (A,B) is stabilizable.

A steady-state $\left(x_{s}, u_{s}\right)$ of system (1) can be parameterized by the parameter $\theta \in \mathbb{R}^{n_{\theta}}[11]$ :

$$
\left[\begin{array}{l}
x_{s} \\
u_{s}
\end{array}\right]=\left[\begin{array}{l}
M_{x} \\
M_{u}
\end{array}\right] \theta,
$$

with $M_{x}, M_{u}$ such that $\left((I-A) M_{x}-B M_{u}\right) \theta=0$.

Definition II.2 (Positively invariant (PI) set). A set $S \subseteq$ $\mathbb{R}^{n}$ is a positively invariant (PI) set of system $x^{+}=A(x)$, if $A(x) \in S$ for all $x \in S$.

Definition II.3 (Robust positively invariant (RPI) set). A set $S \subseteq \mathbb{R}^{n}$ is a robust positively invariant (RPI) set of system $x^{+}=A(x)+w$, if $A(x)+w \in S$ for all $x \in S, w \in \mathcal{W}$.

A PI set that contains every closed PI set of $x^{+}=A(x)$ is called a maximal PI set and similarly for the maximal RPI set.

Definition II.4 (Regional ISS [7], [19]). Given an RPI set $\Gamma \subseteq \mathbb{R}^{n}$ with $0 \in \Gamma$, system $x^{+}=A(x)+w$ is Input-toState Stable (ISS) in $\Gamma$ w.r.t. $w$ if there exists a $\mathcal{K} \mathcal{L}$-function $\beta$ and a $\mathcal{K}$-function $\gamma$ such that for all initial states $x(0) \in \Gamma$ and for all disturbance sequences $\mathbf{w} \triangleq\left[w_{j}\right]_{j \geq 0}$ with $w_{j} \in$ $\mathcal{W}:|\phi(j, x(0), \mathbf{w})| \leq \beta(|x(0)|, j)+\gamma\left(\left\|\mathbf{w}_{[j-1]}\right\|_{\infty}\right), \quad \forall j \geq 0$, where $\phi(k, x(0), \mathbf{w})$ denotes the solution of the system $x^{+}=$ $A(x)+w$ and $\left\|\mathbf{w}_{[j-1]}\right\|_{\infty} \triangleq \sup \{|w(t)|, t \geq 0\}$.

Note that the condition for input-to-state stability reduces to that for asymptotic stability if $\mathbf{w}=0$.

The goal is to regulate the state of the system to the origin which can be formulated as the following MPC problem $\mathbb{P}_{N}(x)$ :

Problem II.5. $\mathbb{P}_{N}(x)$ (Nominal MPC problem)

$$
\begin{aligned}
& V_{N}^{*}(x) \triangleq \min _{\mathbf{u}} V_{N}(x, \mathbf{u}) \triangleq \sum_{i=0}^{N-1} l\left(x_{i}, u_{i}\right)+V_{f}\left(x_{N}\right) \\
& \text { s.t. } x_{0}=x \text {, } \\
& x_{i+1}=A x_{i}+B u_{i}, i=0, \ldots, N-1 \text {, } \\
& \left(x_{i}, u_{i}\right) \in \mathbb{X} \times \mathbb{U}, \quad i=0, \ldots, N-1 \text {, } \\
& x_{N} \in X_{f},
\end{aligned}
$$

where $\mathbf{u}=\left[u_{0}, \cdots, u_{N-1}\right]$ denotes the input sequence, the stage cost is defined as $l\left(x_{i}, u_{i}\right) \triangleq\left\|x_{i}\right\|_{Q}^{2}+\left\|u_{i}\right\|_{R}^{2}$, $V_{f}(x) \triangleq\|x\|_{P}^{2}$ is a terminal penalty function, $Q, R$ and $P$ are symmetric positive definite matrices.

$\mathbb{X} \triangleq\left\{x \mid G_{x} x \leq f_{x}\right\}$ and $\mathbb{U} \triangleq\left\{u \mid G_{u} u \leq f_{u}\right\}$ are polytopic constraints on the states and inputs and $X_{f} \subseteq \mathbb{X}$ is a compact terminal target set. Given a control sequence $\mathbf{u}(x)$ at state $x$, the associated state trajectory is $\mathbf{x}(x) \triangleq$ $\left[x_{0}, x_{1}, \cdots, x_{N}\right]$, where $x_{0}=x$ and for each $i, x_{i}=$ $\phi(i, x, \mathbf{u}(x))$.

Problem $\mathbb{P}_{N}(x)$ implicitly defines the set of feasible control sequences $\mathcal{U}_{N}(x)=\left\{\mathbf{u}(x) \mid \mathbf{u}(x) \in \mathbb{U}^{N}, \mathbf{x}(x) \in\right.$ $\left.\mathbb{X}^{N} \times X_{f}\right\}$ and feasible initial states $\mathcal{X}_{N} \triangleq\left\{x \mid \mathcal{U}_{N}(x) \neq \emptyset\right\}$. For a given state $x \in \mathcal{X}_{N}$ the solution of $\mathbb{P}_{N}(x)$ yields the optimal control sequence $\mathbf{u}^{*}(x)$. The implicit optimal MPC control law is then given in a receding horizon fashion by

$$
\kappa(x) \triangleq u_{0}^{*}(x)
$$

Assumption II.6. In the following it is assumed that $V_{f}(\cdot)$ is a Lyapunov function in $X_{f}$ and $X_{f}$ is a PI set for system (1) under the control law $\kappa_{f}(x) \triangleq K x$. These conditions are stated formally as the following two assumptions:

A1: $X_{f} \subseteq \mathbb{X},(A+B K) X_{f} \subseteq X_{f}, K X_{f} \subseteq \mathbb{U}$

A2: $P>0,(A+B K)^{T} P(A+B K)-P=-\left(Q+K^{T} R K\right)$ 
Theorem II.7 (Stability under $\boldsymbol{\kappa}(\boldsymbol{x})$, [13]). If Assumption II.6 holds, then the closed-loop system $x^{+}=A x+B \kappa(x)$ is asymptotically stable with region of attraction $\mathcal{X}_{N}$.

In order to resolve the feasibility issues described in the introduction, a standard soft constrained approach is to relax the state constraints by the introduction of slack variables $\epsilon_{i}$. $G_{x} x_{i} \leq f_{x}+\epsilon_{i}$. The constraint violation is then minimized by including a penalty on $\epsilon_{i}$ in the MPC cost (see e.g. [12]). This soft constrained method that is also frequently used in practice does however not guarantee stability or satisfaction of the constraints even in the nominal case. The stability proof for the considered finite horizon MPC scheme uses the optimal MPC cost as a Lyapunov function [13]. The stability proof fails in the soft constrained case, since through the introduction of the penalties on $\epsilon_{i}$ into the cost function, it can no longer be shown that the optimal cost is a Lyapunov function.

We propose a new soft constrained MPC formulation in the next section, which provides optimality and constraint satisfaction wherever the state constraints can be enforced (Section IV), a stability guarantee in the nominal case (Section V) and input-to-state stability in the presence of additive disturbances (Section VI).

\section{Soft Constrained MPC - Problem SetuP}

Consider the following soft constrained MPC problem $\mathbb{P}_{N}^{s}(x)$ :

Problem III.1. $\mathbb{P}_{N}^{s}(x)$ (Soft constrained MPC problem)

$$
\begin{aligned}
& V_{N}^{s *}(x)=\min _{\mathbf{u}, \theta, \epsilon, \epsilon_{s}} V_{N}^{s}\left(x, \mathbf{u}, \theta, \epsilon, \epsilon_{s}\right) \\
& V_{N}^{s}\left(x, \mathbf{u}, \theta, \epsilon, \epsilon_{s}\right) \triangleq \sum_{i=0}^{N-1} l\left(x_{i}-x_{s}, u_{i}-u_{s}\right)+\left\|\epsilon_{i}\right\|_{S}^{2} \\
&+\rho_{1}\left\|\epsilon_{i}\right\|_{p}+V_{f}\left(x-x_{s}\right)+\left\|\epsilon_{s}\right\|_{S}^{2}+\rho_{1}\left\|\epsilon_{s}\right\|_{p}+\rho_{2}\|\theta\|_{p} \\
& \text { subject to } \quad=x \\
& x_{0}=A x_{i}+B u_{i} \\
& x_{i+1}=\left[\left(M_{x} \theta\right)^{T}\left(M_{u} \theta\right)^{T}\right] \\
& {\left[x_{s}^{T} u_{s}^{T}\right] } \leq f_{x}+\epsilon_{s}+\epsilon_{i} \\
& G_{x} x_{i} \leq f_{u} \\
& G_{u} u_{i} \geq 0 \\
& \epsilon_{i} \in \mathcal{X}_{f}^{s}(\theta) \\
& \epsilon_{s} \leq f_{x}+\epsilon_{s}-F_{\theta} \theta \\
& x_{N}
\end{aligned}
$$

for $i=[0, \cdots, N-1], S$ is a symmetric positive definite matrix, $p \in[1, \infty], \epsilon=\left[\epsilon_{0}, \ldots, \epsilon_{N-1}\right], \mathcal{X}_{f}^{s}(\theta)$ is an invariant ellipse and $M, T, \theta, F_{\theta}$ are defined in the description below.

Problem $\mathbb{P}_{N}^{s}(x)$ implicitly defines the set of feasible control sequences $\mathcal{U}_{N}^{s}(x)=\{\mathbf{u}(x) \mid \exists \theta: \mathbf{u}(x) \in$ $\left.\mathbb{U}^{N}, \phi(N, x, \mathbf{u}(x)) \in \mathcal{X}_{f}^{s}(\theta)\right\}$ and feasible initial states $\mathcal{X}_{N}^{s} \triangleq\left\{x \mid \mathcal{U}_{N}^{s}(x) \neq \emptyset\right\}$.
For a given state $x \in \mathcal{X}_{N}^{s}$ Problem $\mathbb{P}_{N}^{s}(x)$ results in a convex Second Order Cone Program (SOCP) and its solution yields the optimal control sequence $\mathbf{u}^{s *}(x)$. Note that SOCPs can be efficiently solved using e.g. interior-point methods [2]. The implicit optimal soft constrained MPC control law is then given in a receding horizon fashion by

$$
\kappa^{s}(x) \triangleq u_{0}^{s *}(x) .
$$

Problem $\mathbb{P}_{N}^{s}(x)$ is a modification of $\mathbb{P}_{N}(x)$ introducing the following three components:

- In (5h) the terminal constraint is relaxed by allowing the origin to move to any other feasible steady-state of system (1), parameterized by $\theta$ in (2). The terminal state then has to lie in an invariant set given by $\mathcal{X}_{f}^{s}(\theta)$.

- In (5d) all state constraints from 0 to $N-1$ are softened by means of the slack variables $\epsilon_{s}$ and $\epsilon_{i}$ :

By constraint (5i), $\epsilon_{s}$ characterizes the minimum amount of constraint relaxation that is necessary in order to include the terminal set $\mathcal{X}_{f}^{s}(\theta)$ into the softened state constraints.

$\epsilon_{i}$ represents the additional constraint violation of each state $x_{i}$ with respect to the state constraints relaxed by $\epsilon_{s}$.

- Quadratic and $l_{p}$ penalties on the slack variables are included in the cost (4), in order to minimize the constraint violation and ensure the enforcement of the state constraints whenever possible. An $l_{p}$ penalty on the steady-state is used, minimizing the deviation from the origin. In addition the cost now penalizes the deviation from the steady-state instead of the origin.

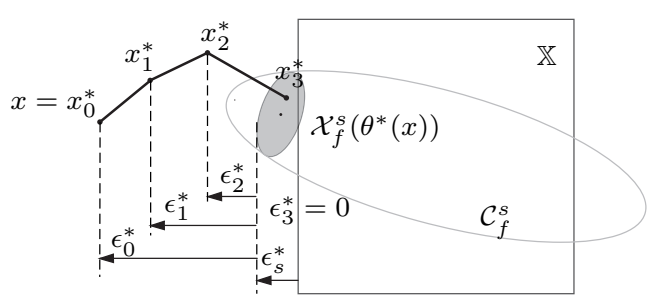

Fig. 1. Illustration of the optimal slack variables $\epsilon_{s}^{*}(x), \epsilon_{i}^{*}(x), i=$ $0,1,2,3$, the terminal set $\mathcal{X}_{f}^{s}\left(\theta^{*}(x)\right)$ and $\mathcal{C}_{f}^{s}$ for an initial state $x$ outside $\mathbb{X}$.

The previously described components are illustrated in Figure 1. The set of states, for which there exists a $\theta$ such that $x \in \mathcal{X}_{f}^{s}(\theta)$ is denoted as $\mathcal{C}_{f}^{s}$ and is further explained in Section III-A. By relaxing the terminal constraint, the soft constrained MPC regulates the state to a feasible steady-state that is simultaneously steered to the origin while minimizing the violation of the state constraints. The use of the slack variable $\epsilon_{s}$ ensures that the terminal state, which is contained in $\mathcal{X}_{f}^{s}(\theta)$, will lie inside the state constraints relaxed by the amount $\epsilon_{s}$ and will not require a further relaxation of the state constraints. As will be shown in Section V, this allows us to show that the optimal cost function is still a Lyapunov function and is hence crucial for proving stability of the proposed soft constrained MPC 
scheme.

The remainder of this section is devoted to a detailed analysis of the two types of soft constraints.

In the following sections we will then demonstrate how the introduction of the previously described components allows us to show that:

1. $\kappa^{s}(x)=\kappa(x)$ wherever the state constraints can be satisfied, i.e. for all $x \in \mathcal{X}_{N}$ (Section IV).

2. The optimal cost function $V_{N}^{s *}(x)$ is a Lyapunov function and the controlled nominal system is asymptotically stable (Section V).

3. The system under additive disturbances is ISS (Section VI).

\section{A. Relaxation of the terminal constraint}

For the relaxation of the terminal set we employ the tracking formulation proposed in [11] where a steady-state, parameterized by $\theta$, is introduced as a decision variable into the optimization problem and the cost then penalizes the distance to the non-zero steady-state instead of the origin as well as the offset from the non-zero steady-state to the origin. The invariant ellipse $\mathcal{X}_{f}^{s}(\theta)$ is obtained from the terminal set for tracking described in [11]. It is defined as the maximal PI set given by the set of states and steadystates and inputs parameterized by $\theta$, such that the control law $u=K\left(x-x_{s}\right)+u_{s}=K x+\left(M_{u}-K M_{x}\right) \theta$ is feasible and stabilizes the nominal system in (1).

Let

$$
\Omega_{f} \triangleq\left\{(x, \theta) \mid\left\|\left[\begin{array}{cc}
T_{f, 1} & T_{f, 2} \\
T_{f, 2}^{T} & T_{f, 3}
\end{array}\right]^{1 / 2}\left[\begin{array}{l}
x \\
\theta
\end{array}\right]\right\|_{2} \leq 1\right\}
$$

be the maximal volume ellipsoidal inner approximation of the maximal PI set.

Remark III.2. Note that a maximal volume ellipsoidal inner approximation $\Omega_{f}$ can be computed by solving a convex linear matrix inequality (LMI) [1].

This implicitly characterizes the set of all feasible steadystates given by $\Theta \triangleq\left\{\theta \mid \exists x: K x+\left(M_{u}-K M_{x}\right) \theta \in\right.$ $\mathbb{U}$ and $\left.(x, \theta) \in \Omega_{f}, M_{u} \theta \in \mathbb{U}\right\}$. For a particular value of $\theta$ the condition $(x, \theta) \in \Omega_{f}$ reduces to $x \in \mathcal{X}_{f}^{s}(\theta)$, with

$$
\begin{array}{r}
\mathcal{X}_{f}^{s}(\theta) \triangleq \quad\{x \mid \\
\left(x-x_{c}(\theta)\right)^{T} T_{f, 1}\left(x-x_{c}(\theta)\right) \\
\left.\leq \theta^{T}\left(T_{f, 2}^{T} T_{f, 1}^{-1} T_{f, 2}-T_{f, 3}\right) \theta\right\}
\end{array}
$$

with $x_{c}(\theta)=-T_{f, 1}^{-1} T_{f, 2} \theta$.

Assumption III.3. By construction $\mathcal{X}_{f}^{s}(\theta)$ is a PI set for system (1) under the control law $\kappa_{f}(x)=K x+\left(M_{u}-\right.$ $\left.K M_{x}\right) \theta$. It therefore satisfies the following assumption:

$$
\text { A3: }\left((A+B K) x+x_{s}\right) \in \mathcal{X}_{f}^{s}(\theta), \kappa_{f}(x) \in \mathbb{U} \forall x \in \mathcal{X}_{f}^{s}(\theta)
$$

This also characterizes the set of all values $x$ for which there exists a feasible steady-state, given by $\mathcal{C}_{f}^{s}=\{x \mid \exists \theta \in \Theta$ : $\left.x \in \mathcal{X}_{f}^{s}(\theta)\right\}$, which can be seen as an enlarged terminal set.

\section{B. Slack variables $\epsilon_{s}$}

We now explain the crucial item in the proposed soft constrained scheme, the use of the slack variables $\epsilon_{s}$ and $\epsilon_{i} . \epsilon_{s}$ represents the amount of constraint relaxation that is necessary in order to include the terminal constraint $\mathcal{X}_{f}^{s}(\theta)$ in (8) for a particular value of $\theta$ into the relaxed state constraints, i.e.

$$
G_{x} x \leq f_{x}+\epsilon_{s} \forall x \in \mathcal{X}_{f}^{s}(\theta)
$$

Lemma III.4. Let $\Omega_{f}$ be the ellipsoidal PI set in (7). Then condition (9) corresponds to constraint (5i)

with $T=T_{f, 2}^{T} T_{f, 1}^{-1} T_{f, 2}-T_{f, 3}$,

$c=\operatorname{diag}\left(G_{x} T_{f, 1}^{-1} G_{x}^{T}\right)$ and $F_{\theta}=G_{x} T_{f, 1}^{-1} T_{f, 2}$.

Proof. Consider the variable transformation $y=T_{f, 1}^{1 / 2}(x-$ $x_{c}(\theta)$ ), with $x_{c}(\theta)=-T_{f, 1}^{-1} T_{f, 2} \theta$. Then condition (9) is equivalent to requiring that the ellipsoid $y^{T} y \leq \theta^{T} T \theta$ is contained in the polytope $G_{x} T_{f, 1}^{-1 / 2} y \leq f_{x}-G_{x} x_{c}(\theta)$, which can be expressed by the following condition [1]

$$
\left(G_{x, j} T_{f, 1}^{-1} G_{x, j}^{T}\right)\left\|T^{1 / 2} \theta\right\|_{2} \leq f_{x, j}+\epsilon_{s, j}-G_{x, j} x_{c}(\theta), \forall j,
$$

and corresponds to (5i).

$\epsilon_{i}$ in (5h) represents the additional constraint violation of each state $x_{i}$ with respect to the state constraints relaxed by $\epsilon_{s}$. Let $\epsilon_{N}$ be the slack variable of the terminal state defined by $G_{x} x_{N} \leq f_{x}+\epsilon_{s}+\epsilon_{N}$. Since $x_{N} \in \mathcal{X}_{f}^{s}(\theta)$ it follows from this and (5d), that $\epsilon_{N}=0$, which will be necessary for proving that the cost function is a Lyapunov function in Section V.

\section{Optimality in $\mathcal{X}_{N}$}

In this section we can now show that the behavior of the soft constrained control law corresponds to the hard constrained one, wherever the state constraints can be satisfied. The constraint violations $\epsilon_{s}$ and $\epsilon_{i}$ are penalized in the cost. Two types of penalty functions are included, quadratic and $l_{p}$-norm penalties, in order to allow for flexibility in modeling the soft constraints. While the quadratic penalty may be preferred for tuning purposes, the $l_{p}$-norm is included in order to allow for exact penalties. It is well-known that, when the weights on the $l_{p}$-norms are sufficiently large and there exists a feasible solution to the hard-constraint problem $\mathbb{P}_{N}(x)$ then the solution to the soft constrained problem $\mathbb{P}_{N}^{s}(x)$ corresponds to the solution of the hard-constraint problem [5], [12]. An $l_{p}$-norm is also used for penalizing the deviation of the steady-state from the origin in order to enforce the origin as the target steady-state if it is feasible [4].

Consider the following optimization problem $\mathbb{P}_{N}^{h}(x)$, enforcing all state constraints as hard constraints:

$$
\begin{gathered}
\min V_{N}^{s}\left(x, \mathbf{u}, \theta, \epsilon, \epsilon_{s}\right) \\
\text { s.t. }(5 \mathrm{a})-(5 \mathrm{i}), \\
\left\|\epsilon_{s}\right\|_{p}=0
\end{gathered}
$$




$$
\begin{aligned}
& \left\|\epsilon_{i}\right\|_{p}=0, i=0, \ldots, N-1, \\
& \|\theta\|_{p}=0 .
\end{aligned}
$$

Note that the optimizer of $\mathbb{P}_{N}^{h}(x)$ corresponds to the optimizer of $\mathbb{P}_{N}(x)$.

Theorem IV.1 (Optimality in $\mathcal{X}_{N}$ [5]). Consider problem $\mathbb{P}_{N}^{h}(x)$. Let $\lambda_{s s}^{*}(x)$ denote the optimal Lagrange multiplier corresponding to constraint (10e) and $\lambda_{\epsilon}^{*}(x)$ the optimal Lagrange multipliers corresponding to the equality constraints (10d) and (10c) at a given state $x \in \mathcal{X}_{N}$. Let $\kappa^{s}(x)$ be the optimal soft constrained control law in (6) and $\kappa^{*}(x)$ the optimal hard constrained control law in (3). If $\rho_{1} \geq \lambda_{s s}^{*}(x)$ and $\rho_{2} \geq \lambda_{\epsilon}^{*}(x)$ for all $x \in \mathcal{X}_{N}$, then $\kappa^{s}(x)=\kappa^{*}(x)$ for all $x \in \mathcal{X}_{N}$.

A lower bound for $\rho_{1}$ and $\rho_{2}$ can e.g. be obtained by computing the optimal Lagrange multipliers parametrically for all $x \in \mathcal{X}_{N}^{s}$. Since this requires the solution of a parametric program and can be computationally infeasible for large systems, a more conservative bound for all values of $x$ can be computed by directly upper bounding the Lagrange multipliers, see e.g. [4] and the references therein.

\section{NOMinal Stability}

After having shown that the soft constrained scheme preserves optimality when possible, we now prove that the resulting optimal control law $\kappa^{s}(x)$ asymptotically stabilizes the nominal system in (1) in an enlarged invariant set $\mathcal{X}_{N}^{s}$. For this, we first show that the optimal cost function $V_{N}^{s *}$ is a Lyapunov function.

Lemma V.1. Consider Problem $\mathbb{P}_{N}^{s}(x)$ under Assumptions II.1-III.3. Let $\mathbf{u}^{s *}(x)=\left[u_{0}^{s *}(x), \ldots, u_{N-1}^{s *}(x)\right], \theta^{*}(x)$, $\epsilon^{*}(x)=\left[\epsilon_{0}^{*}(x), \ldots, \epsilon_{N-1}^{*}(x)\right], \epsilon_{s}^{*}$ be the optimizer of $\mathbb{P}_{N}^{s}(x)$ for some $x \in \mathcal{X}_{N}^{s}, \mathbf{x}^{s *}(x)=\left[x, x_{1}^{s *}(x), \ldots, x_{N}^{s *}(x)\right]$ the associated state trajectory and $x_{s}^{s *}(x)=M_{x} \theta^{*}(x), u_{s}^{s *}(x)=$ $M_{u} \theta^{*}(x)$ the steady-state defined by $\theta^{*}(x)$. Then for $x^{+}=$ $A x+B u_{0}^{s *}(x)$

$u_{s h i f t}=\left[u_{1}^{s *}(x), \ldots, u_{N-1}^{s *}(x), K\left(x_{N}^{s *}(x)-x_{s}^{s *}(x)\right)+u_{s}^{s *}(x)\right]$

is feasible for $\mathbb{P}_{N}^{s}\left(x^{+}\right)$and

$$
V_{N}^{s *}\left(x^{+}\right)-V_{N}^{s *}(x) \leq-l\left(x-x_{s}^{s *}(x), u_{0}^{s *}(x)-u_{s}^{s *}(x)\right) .
$$

Proof. The states and slack variables associated with $u_{\text {shift }}$ in (11) are $\mathbf{x}_{\text {shift }}(x)=\left[x_{1}^{s *}, \ldots, x_{N}^{s *},(A+B K)\left(x_{N}^{s *}-x_{s}^{s *}\right)+x_{s}^{s *}\right]$ and $\epsilon_{\text {shift }}(x)=\left[\epsilon_{1}^{*}, \ldots, \epsilon_{N-1}^{*}, 0\right], \epsilon_{s}^{*}$. Feasibility of $u_{\text {shift }}(x)$ for $\mathbb{P}_{N}^{s}\left(x^{+}\right)$then follows from feasibility of $\mathbf{u}^{s *}(x), \theta^{*}(x)$ at $x$ and positive invariance of the terminal set. $\epsilon_{N}^{*}=0$ follows from the fact that $x_{N} \in \mathcal{X}_{f}^{s}\left(\theta^{*}(x)\right)$ and together with the definition of the sequences and Assumptions II.6 and III.3 provides that $V_{N}^{s}\left(x^{+}, u_{\text {shift }}, \theta^{*}, \epsilon_{\text {shift }}, \epsilon_{s}^{*}\right)-V_{N}^{s *}(x) \leq$ $-l\left(x-x_{s}^{s *}(x), u_{0}^{s *}(x)-u_{s}^{s *}(x)\right)$. (12) then follows from $V_{N}^{s *}\left(x^{+}\right) \leq V_{N}^{s}\left(x^{+}, u_{\text {shift }}, \theta^{*}, \epsilon_{\text {shift }}, \epsilon_{s}^{*}\right)$.
In order for $V_{N}^{s *}(x)$ to be a Lyapunov function and prove asymptotic convergence to the origin, we now need to show that $l\left(x-x_{s}^{*}(x), u_{0}^{s *}(x)-u_{s}^{s *}(x)\right)=0$ implies that $\|x\|=0$.

Lemma V.2. If at a given state $x$ the optimal solution to $\mathbb{P}_{N}^{s}(x)$ is such that $\left\|x-x_{s}^{s *}(x)\right\|=0$, then $\|x\|=0$.

Proof. We first sketch the proof that is a modification of the proof of Lemma 3 in [11], which is proven by contradiction and then extend it to the case considered here.

In the first part of the proof it is shown that for every feasible steady-state $\left(x_{s}, u_{s}\right)$ parameterized by $\theta$ there exists a steadystate $\left(\alpha x_{s}, \alpha u_{s}\right)$ or $\alpha \theta$ with $\alpha \in(0,1)$ such that $x_{s} \in$ $\mathcal{X}_{N}^{s}(\alpha \theta)$. Therefore the control sequence $\mathbf{u}_{\alpha}\left(x_{s}\right)$ generated by the law $u_{i}=K\left(x_{i}-\alpha x_{s}\right)+\alpha u_{s}$ with $x_{0}=x_{s}$ is feasible at $x_{s}$. Let $\mathbf{x}_{\alpha}\left(x_{s}\right)$ be the state sequence corresponding to $\mathbf{u}_{\alpha}$. In the second part of the proof it is shown that, if the current state is $x_{s}$, then the cost to move to $\alpha x_{s}$ applying $\mathbf{u}_{\alpha}\left(x_{s}\right)$ is in fact smaller than the cost of staying at $x_{s}$ by applying $u_{s}$ over the entire horizon and therefore staying at $x_{s}$ cannot be the optimal solution. It was shown that $V_{N}\left(x_{s}, \mathbf{u}_{\alpha}\right)+\rho_{2}\|\alpha \theta\| \leq V_{N}\left(x_{s}, \mathbf{u}_{s}\right)+\rho_{2}\|\theta\|$, where $V_{N}$ is the MPC cost without slack variables in II.5 and $\mathbf{u}_{s}=$ $\left[u_{s}, \ldots, u_{s}\right]$.

In order to prove that this extends to the soft constrained approach we need to show that also

$V_{N}^{s}\left(x_{s}, \mathbf{u}_{\alpha}, \alpha \theta, \epsilon_{\alpha}, \epsilon_{s, \alpha}\right)<V_{N}^{s}\left(x_{s}, \mathbf{u}_{s}, \theta, \epsilon, \epsilon_{s}\right)$ or

$\sum_{i=0}^{N-1}\left\|\epsilon_{i, \alpha}\right\|_{S}^{2}+\rho_{1}\left\|\epsilon_{i, \alpha}\right\|_{p}+\left\|\epsilon_{s, \alpha}\right\|_{S}^{2}+\rho_{1}\left\|\epsilon_{s, \alpha}\right\|_{p}$

$\leq \sum_{i=0}^{N-1}\left\|\epsilon_{i}\right\|_{S}^{2}+\rho_{1}\left\|\epsilon_{i}\right\|_{p}+\left\|\epsilon_{s}\right\|_{S}^{2}+\rho_{1}\left\|\epsilon_{s}\right\|_{p}$,

where $\epsilon_{s}$ and $\epsilon_{i}$ are the minimum slacks corresponding to $\theta$ and a state sequence $\mathbf{x}_{s}=\left[x_{s}, \ldots, x_{s}\right]$ and $\epsilon_{s, \alpha}$ and $\epsilon_{i, \alpha}$ are the minimum slacks corresponding to $\alpha \theta$ and the state sequence $\mathbf{x}_{\alpha}\left(x_{s}\right)$ defined by the constraints (5d) and (5i). This is achieved by the fact that $\epsilon_{s}$ and $\epsilon_{s, \alpha}$ characterize the state relaxation necessary in order to include the terminal sets $\mathcal{X}_{N}^{s}(\theta)$ and $\mathcal{X}_{N}^{s}(\alpha \theta)$, respectively, into the softened state constraints. Since $x_{s} \in \mathcal{X}_{N}^{s}(\theta)$ and also $x_{s} \in \mathcal{X}_{N}^{s}(\alpha \theta), \epsilon_{i}$ and $\epsilon_{i, \alpha}$, representing the extra constraint violation on top of $\epsilon_{s}$ and $\epsilon_{s, \alpha}$, are zero.

We further have that $\epsilon_{s, \alpha}=\left[c\left\|T^{1 / 2} \alpha \theta\right\|_{2}-f_{x}+F_{\theta} \alpha \theta\right]_{+}=$ $\left[\alpha\left(c\left\|T^{1 / 2} \theta\right\|_{2}-f_{x}+F_{\theta} \theta\right)-(1-\alpha) f_{x}\right]_{+} \leq \epsilon_{s}$. Therefore $\left\|\epsilon_{s, \alpha}\right\|_{p} \leq\left\|\epsilon_{s}\right\|_{p},\left\|\epsilon_{s, \alpha}\right\|_{S}^{2} \leq\left\|\epsilon_{s}\right\|_{S}^{2}$, concluding the proof.

These results allow us to prove asymptotic stability of the closed loop system under the soft constrained control law:

Theorem V.3 (Asymptotic Stability under $\kappa^{s}(x)$ ). The closed loop system $x^{+}=A x+B \kappa^{s}(x)$ is asymptotically stable with region of attraction $\mathcal{X}_{N}^{s}$.

Proof. Lemma V.1 together with Lemma V.2 show that $V_{N}^{s *}(x)$ is a Lyapunov function in $\mathcal{X}_{N}^{s}$, i.e. $V_{N}^{s *}\left(x^{+}\right)-$ $V_{N}^{s *}(x) \leq-\gamma(\|x\|)$, for some $\mathcal{K}$-class function $\gamma$ and $x^{+}$in (1), therefore $\lim _{t \rightarrow \infty} x(t)=0$ and the closed-loop system is asymptotically stable.

The soft constrained formulation $\mathbb{P}_{N}^{s}(x)$ enlarges the feasible set compared to $\mathbb{P}_{N}(x)$ since $\mathcal{X}_{N} \subseteq \mathcal{X}_{N}^{s}$ and by choosing the prediction horizon accordingly it can be chosen to cover 
any polytopic region of interest, e.g. a known upper bound on the state values, up to the maximum stabilizable set for the input-constrained system.

Corollary V.4. Let $\beta \mathbb{X}$, with $\beta>1$, be a scaling of the state constraints and $\mathcal{X}_{\infty}:=\left\{x_{0} \mid \exists u_{i} \in \mathbb{U}: x_{i+1}=A x_{i}+\right.$ $\left.B u_{i}, \lim _{j \rightarrow \infty} x_{j}=0, \forall i \geq 0\right\}$ the set of all stabilizable states. There exists a finite prediction horizon $\bar{N}$ such that $\mathcal{X}_{\bar{N}}^{s} \supseteq \beta \mathbb{X} \cap \mathcal{X}_{\infty}$

\section{Robust Stability PRoperties}

In practice, model uncertainties or external disturbances cause a deviation from the nominal system dynamics in (1). The question is then if the control law that was designed for the nominal system model is still stabilizing for the uncertain system, or so-called robustly stable. This issue has been studied in the literature, see e.g. [6], [10] and the references therein. While in general, nominal MPC controllers can have zero robust stability margin, it was shown that particularly for linear systems the nominal MPC controller often offers robust stability in an RPI set under certain assumptions on the MPC problem setup and for a sufficiently small bound on the disturbance size [6], [10].

Another possibility to handle uncertainties is to take them explicitly into account using robust MPC schemes that provide robust stability by changing the problem formulation and tightening the constraints, e.g. min-max MPC or tube-based approaches (see e.g. [10], [13], [14] and the references herein).

The disadvantage of robust MPC methods is however, that a bound on the disturbance size is assumed and the problem is designed for the worst-case disturbance. If the disturbance is then significantly smaller than the worst-case bound for most of the time, the solutions can be highly conservative. If, on the other hand, the disturbance exceeds the expected bound, then the robust MPC problem is infeasible and cannot provide a control input.

When using a nominal MPC scheme, no knowledge on the size of the uncertainty is required for the computation of the controller and it may even be able to take advantage of a disturbance in the right direction. Conservatism is however introduced when analyzing the RPI set, where robust stability of a nominal MPC scheme can be guaranteed, since it is based on a particular bound on the disturbance size. In the presence of hard constraints, the RPI set may be prohibitively small for the disturbance size of interest. The idea is therefore that by using the proposed soft constrained approach robust stability can be guaranteed in a much bigger RPI set due to the fact that state constraints can be relaxed. It is however important to note that, while stability is formally only guaranteed within the RPI set, the control law is defined everywhere in a large feasible set (see Corollary V.4) and may still be stabilizing the uncertain system for a variety of disturbance signals. Note that no RPI sets need to be computed in order to apply the proposed method.

The robust stability properties of the proposed soft constrained MPC scheme are analyzed in the following.

Assume that the system is subject to an additive uncertainty:

$$
x^{+}=A x+B u+w,
$$

where $w$ is a bounded disturbance that is contained in a convex and compact set $\mathcal{W} \subset \mathbb{R}^{n}$ that contains the origin.

Because of the disturbance, the shifted sequence $\mathbf{u}_{\text {shift }}$ in (11) is no longer feasible for $\mathbb{P}_{N}^{s}\left(x^{+}\right)$. For all $x \in \mathcal{X}_{N}^{s}$ there does however exist a feasible solution to $\mathbb{P}_{N}^{s}(x)$ and inputto-state stability can be shown in an RPI set $\mathcal{X}_{\mathcal{W}} \subset \mathcal{X}_{f}^{s}(x)$ (see Theorem VI.2). It is given by the robust invariant set for the controlled uncertain system $x^{+}=A x+B \kappa^{s}(x)+w$, where $\kappa^{s}(x)$ is the optimal soft constrained MPC control law in (6):

$$
A x+B \kappa^{s}(x)+w \in \mathcal{X}_{\mathcal{W}} \forall x \in \mathcal{X}_{\mathcal{W}}, w \in \mathcal{W} .
$$

In order to show that the uncertain system in (13) is robustly stable under the nominal control law we make use of the following result:

Lemma VI.1 (Continuity of $\boldsymbol{V}_{\boldsymbol{N}}^{\boldsymbol{s}}(\boldsymbol{x})$ ). Consider the optimization problem $\mathbb{P}_{N}^{s}(x)$. The optimal value function $V_{N}^{s *}(x)$ is continuous on $\mathcal{X}_{N}^{s}$.

Proof. The value function $V_{N}^{s}\left(x, \mathbf{u}, \theta, \epsilon, \epsilon_{s}\right)$ in (4) and the constraints (5a)-(5i) are convex. Convexity of the optimal cost function $V_{N}^{s *}(x)$ can then be shown using the fact that for any convex set $\mathrm{F}$ in $\left.\mathbb{R}^{p+1}, f(x)=\inf \{\mu \mid(x, \mu) \in F)\right\}$ is a convex function in $\mathbb{R}^{p+1}$ (Theorem 5.3 in [17]) and the fact that the projection of a convex set is convex [17]. The result then follows from the fact that every convex function is continuous on its domain [17].

This allows us to prove ISS of the uncertain system controlled by $\kappa^{s}(x)$ in (6).

Theorem VI.2 (ISS under $\boldsymbol{\kappa}^{\boldsymbol{s}}(\boldsymbol{x})$ ). The closed loop system $x^{+}=A x+B \kappa^{s}(x)+w$ is ISS w.r.t to $w \in \mathcal{W}$ with region of attraction $\mathcal{X}_{\mathcal{W}}$.

Proof. From Lemma V.1, V.2 and VI.1 it follows that $V_{N}^{s *}(x)$ is a continuous Lyapunov function and hence there exists a $\mathcal{K}$-class function $\sigma_{V}$, such that $\left|V_{N}^{s *}(y)-V_{N}^{s *}(x)\right| \leq \sigma_{V}(\| y-$ $x \|)$ as well as a $\mathcal{K}$-class function $\gamma$ such that $V_{N}^{s *}\left(x^{+}\right)-$ $V_{N}^{s *}(x) \leq-\gamma(\|x\|)$. It follows from these facts that

$$
\begin{aligned}
V_{N}^{s *}\left(x^{+}\right)-V_{N}^{s *}(x) \\
=V_{N}^{s *}\left(A x+B \kappa^{s}(x)+w\right)-V_{N}^{s *}\left(A x+B \kappa^{s}(x)\right) \\
\quad+V_{N}^{s *}\left(A x+B \kappa^{s}(x)\right)-V_{N}^{s *}(x) \\
\leq\left|V_{N}^{s *}\left(A x+B \kappa^{s}(x)+w\right)-V_{N}^{s *}\left(A x+B \kappa^{s}(x)\right)\right| \\
\quad-l\left(x-x_{s}^{s *}(x), u-u_{s}^{s *}(x)\right) \\
\leq \sigma_{V}(\|w\|)-\gamma(\|x\|) .
\end{aligned}
$$

Then $V_{N}^{s *}(x)$ is an ISS-Lyapunov function and the closedloop system is ISS [7]. 
The uncertain system controlled by the control law resulting from the soft constrained MPC problem $\mathbb{P}_{N}^{s}(x)$ is hence robustly stable against sufficiently small disturbances. Since the RPI set $\mathcal{X}_{\mathcal{W}}$ depends on $\mathcal{W}$, the size of the disturbances and the corresponding region for which stability can be formally guaranteed depend on the particular system of interest.

\section{NUMERICAL EXAMPLE}

The presented results are demonstrated in the following section using a numerical example. All set computations were carried out using the YALMIP toolbox [9] and the MPT toolbox [8].

Consider the following system:

$$
x_{i+1}=\left[\begin{array}{cc}
1.05 & 1 \\
0 & 1
\end{array}\right] x_{i}+\left[\begin{array}{c}
1 \\
0.5
\end{array}\right] u_{i}
$$

The system has eigenvalues at $s_{1}=1.05, s_{2}=1$ and is hence unstable. The prediction horizon was chosen to $N=5$, the constraints on the states and control inputs to $\|x\|_{\infty} \leq 5$ and $\|u\|_{\infty} \leq 1, Q=I, R=1$ and $S=I$. The terminal cost function $V_{f}(x)$ is taken as the unconstrained infinite horizon optimal value function for the nominal system with $P=\left[\begin{array}{lll}1.9119 & 0.2499 \\ 0.2499 & 2.6510\end{array}\right]$ and $\kappa_{f}(x)=K x$ is the corresponding optimal LQ controller. The exact penalty multipliers were chosen to $\rho_{1}=\rho_{2}=50$ which was observed to provide optimality in $\mathcal{X}_{N}$ as defined in Theorem IV.1.

First, the feasible set $\mathcal{X}_{5}^{s}$ and the terminal set $\mathcal{X}_{f}^{s}$ for the soft constrained approach $\mathbb{P}_{N}^{s}(x)$ are illustrated and compared with the feasible set $\mathcal{X}_{5}(x)$ and the terminal set $\mathcal{X}_{f}$ for the hard constraint problem $\mathbb{P}_{N}(x)$ in Figure 2, which demonstrates that the soft constrained approach significantly enlarges the feasible set. In addition we plot a state set of interest taken as $2 \mathbb{X} \cap \mathcal{X}_{\infty}$. It can be seen that for a horizon of $N=5$ the set is not included in the feasible set $\mathcal{X}_{5}^{s}$. If we however prolong the horizon to $N=8$, then the set of interest is included in $\mathcal{X}_{8}^{s}$.

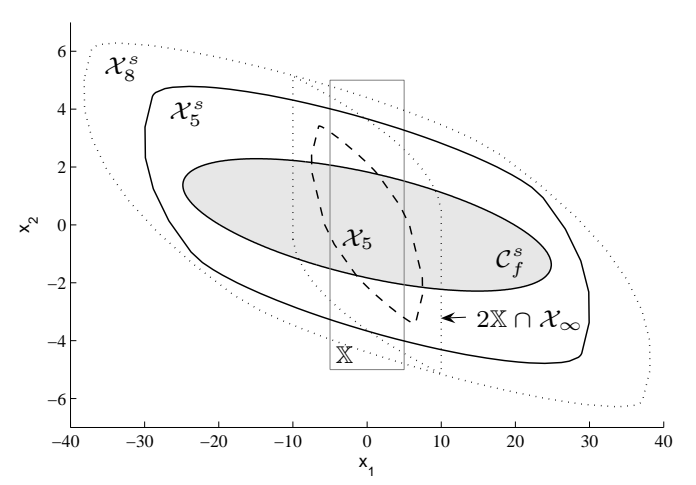

Fig. 2. Feasible and terminal sets for the soft constrained approach for $N=$ 5 in comparison with the feasible and terminal set of the hard constraint problem. The state set of interest $2 \mathbb{X} \cap \mathcal{X}_{\infty}$ can be covered by $\mathcal{X}_{8}^{s}$.
We now analyze the robustness properties of the example system (15) under the soft constrained control law. Figure 3 shows the size of the RPI sets $\mathcal{X}_{\mathcal{W}}$ for two bounds $\mathcal{W}_{\bar{w}} \triangleq$ $\left\{w \mid\|w\|_{\infty} \leq \bar{w}\right\}$, for $\bar{w} \in\{0.1,0.25\}$. Note that for a tube-based approach the feasible set is always a subset of $\mathcal{X}_{N}$. This demonstrates the advantage of the soft constrained approach, where stability can be guaranteed in the RPI set $\mathcal{X}_{\mathcal{W}_{0.25}} \supseteq \mathcal{X}_{N}$. In addition, a closed-loop trajectory starting at $x(0)=[20,-1]^{T}$ under a sequence of extreme disturbances with $w(t)= \pm 0.25, t \geq 0$ is shown and demonstrates that the system is stable and does not leave the RPI set $\mathcal{X}_{\mathcal{W}_{0.25}}$.

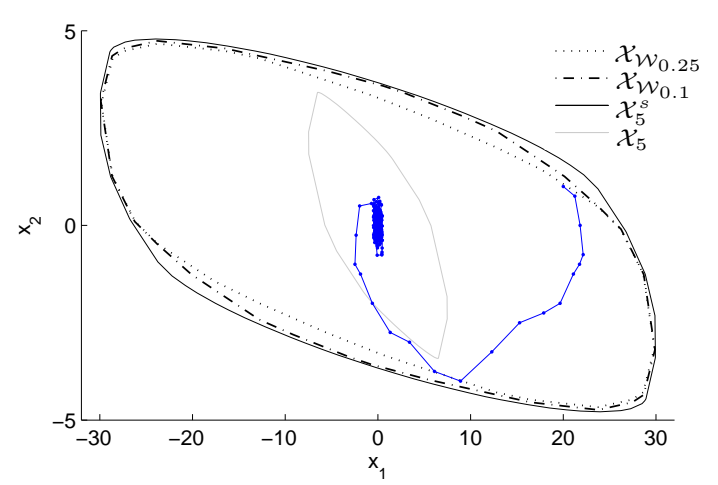

Fig. 3. Feasible set and RPI sets for $\bar{w} \in\{0.1,0.25\}$ together with a closed-loop trajectory starting at $x(0)=[20,-1]^{T}$ under a sequence of extreme disturbances.

\section{REFERENCES}

[1] S. Boyd, L. E. Ghaoui, E. Feron, and V. Balakrishnan, "Linear Matrix Inequalities in System and Control Theory," in Volume 15 of Studies in Applied Mathematics. Society for Industrial and Applied Mathematics (SIAM), 1994.

[2] S. Boyd and L. Vandenberghe, Convex Optimization. Cambridge University Press, 2004.

[3] N. de Oliveira and L.T.Biegler, "Constraint Handling and Stability Properties of Model-Predictive Control," AIChE Journal, vol. 40, pp. 1138-1155, 1994.

[4] A. Ferramosca, D. Limon, I. Alvarado, T. Alamo, and E. Camacho, "MPC for tracking with optimal closed-loop performance," Automatica, vol. 45, pp. 1975-1978, 2009.

[5] R. Fletcher, Practical Methods of Optimization, 2nd ed. John Wiley and Sons, New York, 1987.

[6] G. Grimm, M. Messina, S. Tuna, and A. Teel, "Examples when nonlinear model predictive control is nonrobust," Automatica, vol. 40, pp. 1729-1738, 2004.

[7] Z.-P. Jiang and Y. Wang, "Input-to-state stability for discrete-time systems," Automatica, vol. 37, pp. 857-869, 2001.

[8] M. Kvasnica, P. Grieder, M. Baotic, and M. Morari, Multi-Parametric Toolbox (MPT), Mar. 2004, pp. 448-462.

[9] J. Löfberg, "YALMIP : A Toolbox for Modeling and Optimization in MATLAB," in Proceedings of the CACSD Conference, Taipei, Taiwan, 2004.

[10] D. Limon, T. Alamo, D. Raimondo, D. M. de la Peña, J. Bravo, A. Ferramosca, and E. Camacho, "Input-to-State Stability: A Unifying Framework for Robust Model Predictive Control," Lecture Notes in Control and Information Sciences, vol. 384, pp. 1-26, 2009.

[11] D. Limon, I. Alvarado, T. Alamo, and E. Camacho, "MPC for tracking piecewise constant references for constrained linear systems," Automatica, vol. 44, pp. 2382-2387, 2008.

[12] J. Maciejowski, Predicive Contorl with Constraints. Prentice Hall, 2000.

[13] D. Mayne, J. Rawlings, C. Rao, and P. Scokaert, "Constrained model predictive control: Stability and optimality," Automatica, vol. 36(6), pp. 789-814, 2000. 
[14] D. Mayne, M. Seron, and S. Rakovic, "Robust model predictive control of constrained linear systems with bounded disturbances," Automatica, vol. 41, pp. 219-234, 2005.

[15] J. Primbs, "A Soft Constraint Approach to Stochastic Receding Horizon Control," in Proc. of the 48th Conf. on Decision and Control, 2007.

[16] J. Rawlings and K. Muske, "The stability of Constrained Receding Horizon Control," IEEE Trans. on Automatic Control, vol. 38, pp. 1512-1516, 1993.

[17] R. Rockafellar, Convex Analysis. Princeton University Press, 1970.

[18] P. Scokaert and J. Rawlings, "Feasibility Issues in Linear Model Predictive Control," AIChE Journal, vol. 45, pp. 1649 - 1659, 1999.

[19] E. Sontag and Y. Wang, "New characterizations of input-to-state stability," IEEE Transactions on Automatic Control, vol. 44, pp. 648654, 1999.

[20] M. Vidyasagar, Nonlinear Systems Analysis, 2nd ed. Prentice Hall, 1993.

[21] A. Zheng and M. Morari, "Stability of Model Predictive Control with Mixed Constraints," IEEE Trans. on Automatic Control, vol. 40, pp. 1818-1823, 1995. 